\title{
V4140 Sgr: A short period dwarf nova with a peculiar behavior ${ }^{\star}$
}

\author{
B. W. Borges and R. Baptista
}

\begin{abstract}
Departamento de Física, Universidade Federal de Santa Catarina, CEP 88040-900, Florianópolis, Brazil e-mail: [bernardo; bap] @astro.ufsc.br
\end{abstract}

Received 21 September 2004 / Accepted 19 March 2005

\begin{abstract}
We report on time series of CCD photometry of V4140 Sgr between 1991 and 2001. The analysis reveals that the object was in the decline from an outburst in 1992 and again in outburst in 2001. The historical light curve collected by amateur astronomers shows low amplitude (1-2 mag) outbursts 5-10 days long, recurrent every 80-90 days, confirming its dwarf nova

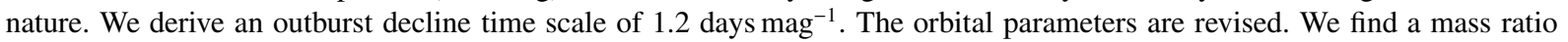
$q=M_{1} / M_{2}=0.125 \pm 0.015$, an inclination $i=80.2^{\circ} \pm 0.5^{\circ}$, and $M_{1}=0.73 \pm 0.08 M_{\odot}$ and $M_{2}=0.09 \pm 0.02 M_{\odot}$, respectively for the primary and secondary star masses. The predicted values for the semi-amplitude of the radial velocity curve of the primary and secondary stars are $K_{1}=55 \pm 7 \mathrm{~km} \mathrm{~s}^{-1}$ and $K_{2}=442 \pm 15 \mathrm{~km} \mathrm{~s}^{-1}$, respectively. Eclipse mapping techniques were applied to data both in quiescence and in outburst to derive accretion disc surface brightness maps. A distance of $d=170 \pm 30 \mathrm{pc}$ is obtained from a method similar to that used to constrain the distance to open clusters. From this distance, disc radial brightness temperature distributions are determined. The temperatures in the quiescent disc vary from $6000 \mathrm{~K}$ in the inner regions to $3000 \mathrm{~K}$ near the outer disc and are flatter than the $T \propto r^{-\frac{3}{4}}$ law for optically thick steady-state disc. The outburst occurs mainly with a significant increase in brightness of the intermediate and outer disc regions. The disc temperatures remain below the critical effective temperature $T_{\text {crit }}$ at all disc radii during outburst. The radial temperature distributions in quiescence and in outburst are significantly different from those of other dwarf novae of similar orbital period. These results cannot be explained within the framework of the disc instability model. We suggest that the small amplitude outbursts of V4140 Sgr are caused by bursts of enhanced mass transfer rate from the secondary star.
\end{abstract}

Key words. accretion, accretion disks - eclipses - stars: binaries: close - stars: individual: V4140 Sgr novae, cataclysmic variables

\section{Introduction}

Dwarf novae are cataclysmic variable stars (CVs) which show recurrent outbursts typically of 2 to 5 mag on time-scales of weeks to months, caused by a sudden increase in the mass accretion rate. SU UMa stars are a subclass of the dwarf novae which exhibit occasional superoutbursts $\sim 0.7$ mag brighter and $\sim 3-5$ times longer than the regular outbursts.

Two models were proposed back in the 70's to explain the cause of the outbursts. In the mass transfer instability model (MTI), the outburst are the response of a high viscosity accretion disc to a burst of matter transferred from the donor star. In the disc instability model (DI), the outbursts are caused by a thermal-viscous instability cycle in the disc, in which an annulus in a low viscosity state disc reaches a critical condition which propagates as a heating wave and progressively switches the disc to a high viscosity state, causing the rapid inward diffusion of the orbiting gas (see Lasota 2001 for a review). According to the DI model, there is a critical effective temperature $T_{\text {crit }}$, below which the disc gas should remain to allow

^ Based on observations made at the Laboratório Nacional de Astrofísica (LNA/MCT), Brazil. the thermal instability to set in, and above which the disc gas should stay while in outburst (e.g. Warner 1995),

$T_{\text {crit }}(r)=7476\left(\frac{r}{R_{L_{1}}}\right)^{-0.105}\left(\frac{M_{1}}{0.75 M_{\odot}}\right)^{-0.15} \mathrm{~K}$

where $R_{L_{1}}$ is the distance from the disc centre to the inner Lagrangian point and $M_{1}$ is the primary mass.

Over the last two decades the DI model became the dominant theory to explain dwarf novae outburst because it could reproduce observed phenomena seemingly much better than the MTI model (e.g., Cannizzo 1993) and because there is a physical mechanism for the disc instability, which has not been the case for the MTI model (Gontikakis \& Hameury 1993; Warner 1995; Lasota 2001). However, the present situation is more complex. Mass transfer variations became an important element of the DI model. Many aspects of dwarf novae outburst can only be reasonably well reproduced by state-of-the-art DI simulations with the inclusion of variations in mass transfer from the secondary star (King \& Cannizzo 1998; Schreiber et al. 2000, 2003; Buat-Ménard et al. 2001). On the other hand, there are still unsolved problems in the DI model (Smak 2000) and recent detailed observations of some dwarf novae are in 
contradiction with the DI model (Hellier et al. 2000; Baptista \& Catalán 2001; Baptista \& Bortoletto 2004).

V4140 Sgr is a relatively faint, eclipsing $\mathrm{CV}(V \simeq 18 \mathrm{mag})$ with a short orbital period $\left(P_{\text {orb }} \simeq 88 \mathrm{~min}\right)$. The eclipses were discovered by Jablonski \& Steiner (1987). The spectroscopy carried out by Mukai et al. (1988) confirmed its classification as a CV and allowed the measurement of a systemic velocity of $\gamma=58 \pm 5 \mathrm{~km} \mathrm{~s}^{-1}$ and a primary radial velocity semi-amplitude of $K_{1}=56 \pm 7 \mathrm{~km} \mathrm{~s}^{-1}$. Baptista et al. (1989, here after BJS) derived the orbital parameters for the object from high-speed photometry covering 25 eclipses. Baptista \& Steiner (1991) applied eclipse mapping techniques to investigate the structure of the accretion disc of V4140 Sgr. Because their data consisted of uncalibrated white light curves, it was not possible to determine the radial brightness distribution from the eclipse maps. Also, because their light curves were normalized to the out-ofeclipse level, they were not able to distinguish observations in quiescence from those in outburst. Downes et al. (2001) lists V4140 Sgr as an SU UMa star, however until recently there was no observations of outbursts or superoutbursts to confirm that.

This paper reports the analysis of time series $B V R$ CCD photometry of V4140 Sgr. Its is organized as follows. Section 2 describes the observations and the data reduction procedures, and Sect. 3 addresses the observations which characterize the dwarf nova behavior of the star. Section 4 presents the data analysis: the revised orbital parameters (Sect. 4.1) and the eclipse mapping analysis of the $B V R$ light curves (Sect. 4.2). The results are presented in Sect. 5 and discussed in Sect. 6. Section 7 summarizes the main conclusions.

\section{The observations and data reduction}

The observations were performed with the $1.6 \mathrm{~m}$ Perkin-Elmer telescope of Laboratório Nacional de Astrofísica (LNA/MCT Brazil) between 1991 and 2001, comprising a total of 39 eclipses. All data were obtained with high-speed CCD photometry in the $B V R$ bands. Observations before 1996 employed a EEV CCD array with $770 \times 1152$ pixels of $22.5 \times 22.5 \mu \mathrm{m}$. The remaining runs were obtained with a blue sensitive, back illuminated EEV CCD with $385 \times 578$ pixels of $22 \times 22 \mu \mathrm{m}$. The plate scale is $\sim 0.6^{\prime \prime}$ pixel $^{-1}$ for both detectors. Table 1 shows the journal of the observations. $\Delta t$ is the integration time and $N$ is the number of points of each run. The last three columns list the phase range of the observed run, the quality of the night sky and the range of seeing (in arcseconds).

The CCD data reduction was done with IRAF $^{1}$ routines and included the usual bias and flat-field corrections, and the automatic identification and removal of cosmic rays. Aperture photometry was carried out with the IRAF APPHOT package. The fluxes were extracted for the variable and for 5 field stars using

\footnotetext{
${ }^{1}$ Image Reduction and Analysis Facility, a general purpose software system for the reduction and analysis of astronomical data. IRAF is written and supported by National Optical Astronomy Observatories (NOAO) in Tucson, Arizona. NOAO is operated by the Association of Universities for Research in Astronomy Inc. (AURA) under cooperative agreement with the National Science Foundation.
}

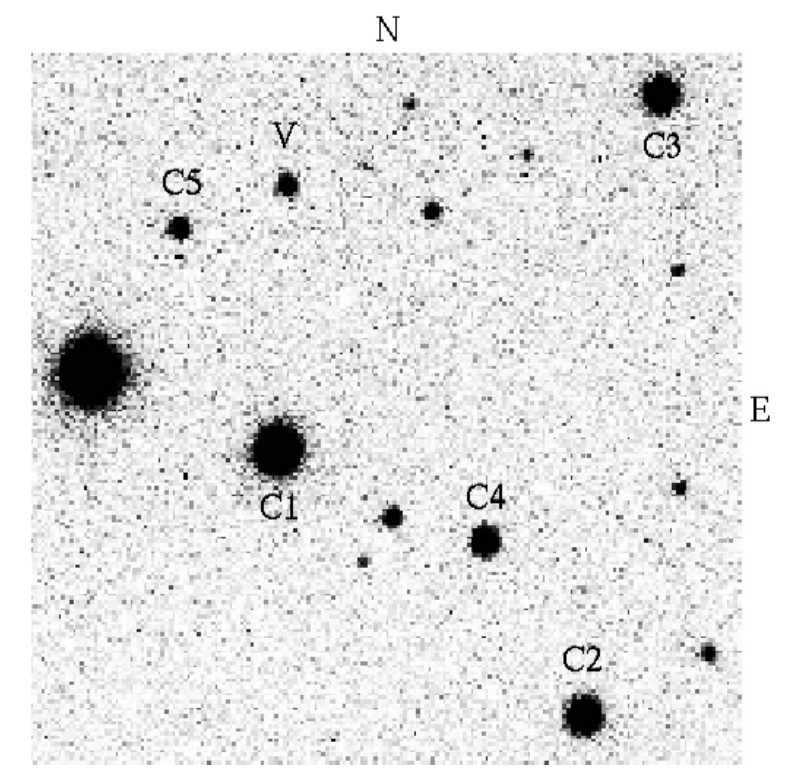

Fig. 1. Image of V4140 Sgr (labeled with V) obtained with a CCD camera in the $V$ band in 1992. The reference star (C1) and the comparison stars $(\mathrm{C} 2$ to $\mathrm{C} 5)$ used in the differential photometry are indicated. The field orientation in North up and East to the right, and its size is $\simeq 2^{\prime} \times 2^{\prime}$.

a 6-pixel radius diaphragm. The sky contribution was estimated from the centroid of the intensity histogram (or weighted mean) of all pixels within an inner radius of 7 pixels and a radial width of 10 pixels, centered in each star. Figure 1 shows an image of the field of V4140 Sgr in which the reference and comparison stars used for the differential photometry are indicated. Time series were constructed by computing the magnitude difference of the variable $(\mathrm{V})$ and other comparison stars (C2 to $\mathrm{C} 5)$ with respect to the reference star $(\mathrm{C} 1)$. The uncertainty in the differential photometry of V4140 Sgr was estimated from the dispersion in the magnitude difference for comparison stars of similar brightness and amounts to $0.06,0.12$ and 0.065 mag respectively in the $B, V$ and $R$ bands. The data were transformed from magnitude to a relative flux scale by assuming a unity flux for the reference star $(\mathrm{C} 1)$. The reference star flux was calibrated from observations of standard stars of Graham (1982) and of blue spectrophotometric standards (Stone \& Baldwin 1983). The photometric calibration program adopts the prescription of Harris et al. (1981) to solve simultaneously all photometric coefficients, including first and second-order extinction (e.g. Jablonski et al. 1994). We used the relations of Lamla (1982) to transform the $B V R$ magnitudes of the reference star to flux units and to flux calibrate the light curves of V4140 Sgr.

\section{V4140 Sgr as a dwarf nova}

The top panel of Fig. 2 displays the visual light curve of V4140 Sgr constructed from observations made by VSNET amateur astronomers (Kato et al. 2004) during 2004. The observations were performed with an unfiltered CCD and reduced to visual magnitude using the $R$ magnitude sequence ${ }^{2}$. The star was observed in outburst three times on a time interval of

\footnotetext{
${ }^{2}$ See http://www. aavso.org (AAVSO homepage)
} 
Table 1. Journal of the observations.

\begin{tabular}{|c|c|c|c|c|c|c|c|c|}
\hline Date (UT) & $\begin{array}{c}\text { Start (HJD) } \\
(2400000+)\end{array}$ & Cycle & Passband & $\begin{array}{l}\Delta t \\
(\mathrm{~s})\end{array}$ & $N$ & $\begin{array}{l}\text { Phase } \\
\text { range }\end{array}$ & $\begin{array}{c}\text { Night } \\
\text { quality }^{\dagger}\end{array}$ & $\begin{array}{c}\text { Seeing } \\
\left({ }^{\prime \prime}\right)\end{array}$ \\
\hline 1991 Jul. 08 & 48446.71492 & 35570 & V & 15,40 & 106 & $-0.15,+0.5$ & B & $1.5-2.5$ \\
\hline " & 48446.75568 & 35571 & $V$ & 15,40 & 133 & $-0.5,+0.45$ & B & \\
\hline$"$ & 48446.81840 & 35572 & $V$ & 15,40 & 115 & $-0.47,+0.26$ & B & \\
\hline 1991 Jul. 09 & 48447.57448 & 35584 & $V$ & 15 & 127 & $-0.16,+0.5$ & B & $1.5-2.5$ \\
\hline " & 48447.61545 & 35585 & $V$ & 15 & 162 & $-0.5,-0.44$ & B & \\
\hline$"$ & 48447.70001 & 35586 & $V$ & 15 & 118 & $-0.12,+0.5$ & B & \\
\hline$"$ & 48447.73816 & 35587 & $V$ & 15 & 122 & $-0.5,+0.35$ & B & \\
\hline$"$ & 48447.81620 & 35588 & $V$ & 15 & 64 & $-0.22,+0.14$ & B & \\
\hline 1991 Jul. 10 & 48448.55692 & 35600 & $R$ & 15 & 99 & $-0.17,+0.38$ & B & $1.5-2.0$ \\
\hline 1992 Jul. 27 & 48831.47956 & 41834 & $R$ & 15,50 & 143 & $-0.34,+0.5$ & $\mathrm{C}$ & $1.3-1.8$ \\
\hline " & 48831.55100 & 41835 & $R$ & 30,50 & 71 & $-0.5,+0.5$ & $\mathrm{C}$ & \\
\hline$"$ & 48831.61239 & 41836 & $R$ & 15,50 & 115 & $-0.5,+0.5$ & $\mathrm{C}$ & \\
\hline$"$ & 48831.67381 & 41837 & $R$ & 15,50 & 111 & $-0.5,+0.5$ & B & \\
\hline$"$ & 48831.73532 & 41838 & $R$ & 15,50 & 119 & $-0.5,+0.43$ & A & \\
\hline 1992 Jul. 28 & 48832.55223 & 41851 & $R$ & 20,50 & 93 & $-0.2,+0.5$ & B & $1.5-2.0$ \\
\hline " & 48832.59537 & 41852 & $R$ & 15,50 & 124 & $-0.5,+0.5$ & B & \\
\hline$"$ & 48832.65663 & 41853 & $R$ & 15,50 & 163 & $-0.5,+0.6$ & B & \\
\hline 1992 Jul. 29 & 48833.53336 & 41867 & V & 20,50 & 67 & $-0.22,+0.28$ & B & $1.3-2.3$ \\
\hline " & 48833.60199 & 41868 & $V$ & 20 & 68 & $-0.1,+0.33$ & B & \\
\hline$"$ & 48833.65432 & 41869 & $V$ & 20,50 & 103 & $-0.25,+0.5$ & B & \\
\hline$"$ & 48833.70111 & 41870 & $V$ & 20,50 & 114 & $-0.5,+0.49$ & B & \\
\hline " & 48833.76214 & 41871 & V & $20,25,50$ & 54 & $-0.5,+0.18$ & B & \\
\hline 1992 Jul. $30^{*}$ & 48834.44980 & 41882 & $R$ & 50 & 1 & -0.3 & B & $1.3-1.5$ \\
\hline " & 48834.45659 & " & $V$ & 25 & 61 & $-0.2,+0.26$ & B & \\
\hline$"$ & 48834.52074 & 41883 & $V$ & 20,50 & 87 & $-0.15,+0.5$ & B & \\
\hline$"$ & 48834.56075 & 41884 & V & 20,50 & 90 & $-0.5,+0.25$ & B & \\
\hline$"$ & 48834.64339 & 41885 & $V$ & 20,50 & 80 & $-0.16,+0.5$ & B & \\
\hline$"$ & 48834.68395 & 41886 & $V$ & 20,50 & 110 & $-0.5,+0.5$ & B & \\
\hline$"$ & 48834.74569 & 41887 & V & 20 & 90 & $-0.49,+0.39$ & B & \\
\hline 1998 Jul. 26 & 51021.71684 & 77488 & $B$ & 20 & 203 & $-0.27,+0.5$ & A & $1.2-1.4$ \\
\hline " & 51021.76387 & 77489 & $B$ & 20 & 295 & $-0.5,+0.34$ & A & \\
\hline 1999 Jul. 12 & 51372.60399 & 83200 & $B$ & 20 & 108 & $-0.25,+0.16$ & A & 1.2 \\
\hline " & 51372.77520 & 83202 & $B$ & 20 & 164 & $-0.47,+0.16$ & A & \\
\hline 1999 Jul. 14 & 51374.79735 & 83236 & $B$ & 20 & 386 & $-0.55,+0.57$ & A & $1.2-1.5$ \\
\hline 2000 Jul. 29 & 51755.67684 & 89436 & $B$ & 20 & 165 & $-0.29,+0.33$ & A & $1.2-1.5$ \\
\hline 2000 Jul. 30 & 51756.47832 & 89449 & $B$ & 20 & 103 & $-0.25,+0.14$ & A & $1.3-1.5$ \\
\hline " & 51756.60539 & 89451 & $B$ & 20 & 92 & $-0.18,+0.17$ & A & \\
\hline 2001 Jun. 25 & 52086.72728 & 94825 & $B$ & 20 & 113 & $-0.20,+0.23$ & A & $1.5-2.0$ \\
\hline 2001 Jun. 28 & 52089.67373 & 94873 & $B$ & 20 & 124 & $-0.23,+0.24$ & B & $1.3-1.8$ \\
\hline " & 52089.80202 & 94875 & $B$ & 20 & 69 & $-0.14,+0.22$ & B & \\
\hline
\end{tabular}

${ }^{\dagger}$ Night Quality: A - photometric; B - good; C - poor (large variations and/or clouds).

* See text (Sect. 3).

7 months. On the 2004 September outburst V4140 Sgr showed superhumps with a period 1.6 per cent longer than the orbital period (Uemura 2004; Uemura et al. 2004, in preparation), thereby confirming that V4140 Sgr is not only a dwarf nova, but also an SU UMa star. The outburst of 2004 June was possibly another superoutburst. The maximum of the 2004 April outburst is fainter than those of the following superoutbursts by $\simeq 0.7 \mathrm{mag}$ - in agreement with the expected for a regular outburst. From Fig. 2 we infer a mean time interval between outbursts of 80-90 days and an outburst duration of 5-10 days, typical of dwarf novae (Warner 1995).

The outbursts of V4140 Sgr have remarkably low amplitude in comparison with bona fide dwarf novae. The maximum of the 2004 Sep superoutburst is only about 2 mag brighter than the typical quiescent level of $V \simeq 18.0 \mathrm{mag}$. Accordingly, the regular outburst (2004 Apr) has an amplitude of only $\simeq 1$ mag. 

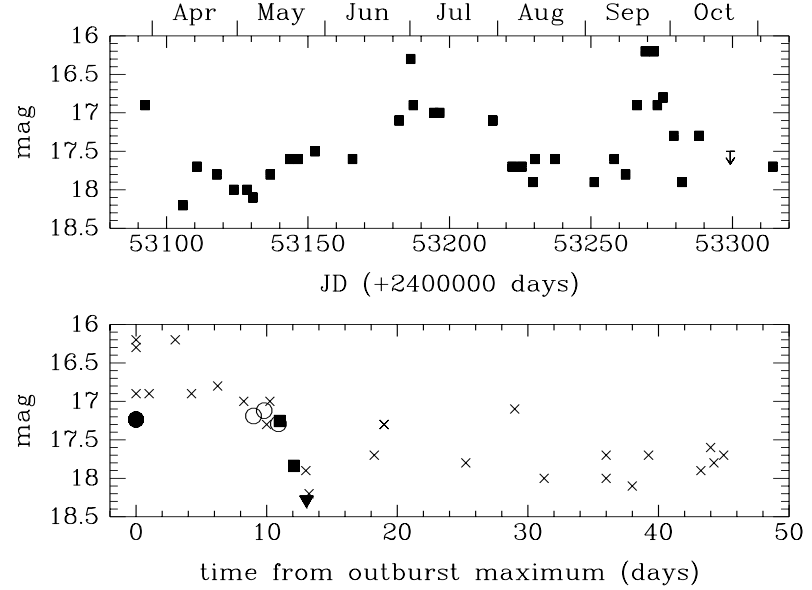

Fig. 2. Top: the visual historical light curve of V4140 Sgr in 2004 constructed from observations made by VSNET amateur astronomers. The epoch of the observations are indicated on the top. Bottom: superimposed outbursts of V4140 Sgr. Crosses indicate visual measurements. Filled symbols are out-of-eclipse mean magnitude of the object from the data of $2001 B$ (circle), $1992 R$ (squares) and $1992 V$ (triangle) (see text). Open circles are from the $V$ measurements of BJS.

These results suggest that the quiescent disc of V4140 Sgr may be significantly brighter than those of the prototype short period dwarf novae OY Car and Z Cha.

The top panel of Fig. 3 shows our $R$ band observations of V4140 Sgr in 1992. The brightness of the star reduced by a factor 2 on a time scale of one night, indicating that the object was caught in the decline from an outburst. The eclipse runs are arbitrarily shown in consecutive binary cycles for a better visualization. Therefore, the real time span between eclipses on different nights is larger than indicated in the figure, except for the truly consecutive cycles. The eclipse in the night of 1991 July 10th (cycle 35600 ) is representative of the quiescent state. The data point in 1992 July 30th (cycle 41 882) is from a single exposure in the $R$ band taken outside of eclipse (orbital phase -0.3 ) and is consistent with the trend in brightness decline inferred from the observations of the previous nights. The object was also caught in outburst in 2001, from observations in the $B$ band. The lower panel of Fig. 3 shows the brightness increase of V4140 Sgr in 2001 June in comparison to observations done in a previous year, when the object was in quiescence. The object shows brightness variations by a factor of $\simeq 2.5$ in the two observed outbursts, confirming that the normal outbursts have small amplitude $(\Delta \mathrm{mag}=1 \mathrm{mag})$. In both cases, the outburst eclipses are relatively shallow, with a depth comparable to that in quiescence, indicating that the brightness increase is mainly from the outer and only partially eclipsed disc regions.

The lower panel of Fig. 2 presents the superimposed outbursts of V4140 Sgr. We use the decline branch of the outburst to align the incomplete outbursts in time and we set the zero time as that corresponding to the maximum brightness of those outburst with full observing coverage. Crosses represent VSNET observations (upper panel of Fig. 2) in visual magnitude. Filled symbols show measurements of the out-of-eclipse mean magnitude of our data: $B$ in outburst in 2001 (circle), $R$ (square) and $V$ (triangle) in decline from the 1992 outburst
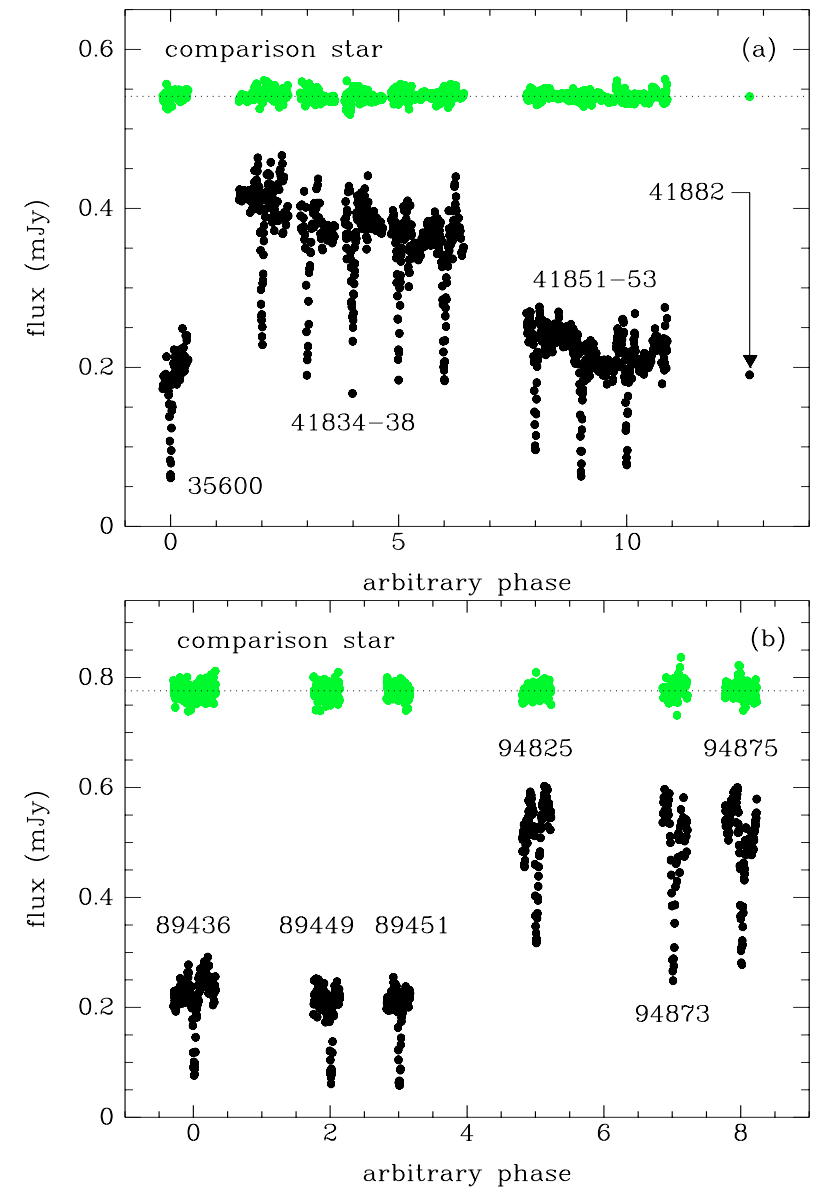

Fig. 3. a) $R$ band observations of V4140 Sgr in the decline from an outburst in 1992 July. The labels indicate the cycles of the observations (see Table 1, for details). The only point in 1992 July 30th refer to a single $R$ band image at that night. The gray points show the light curve of a field comparison star. b) $B$ band observations of V4140 Sgr in outburst in 2001 June in comparison to the quiescence data of 2000 July. The separation of the light curves in the $x$-axis is arbitrary in both panels and was chosen for visualization purposes only.

(see Table 1 for details). The $V$ and $R$ points were spaced using the mean interval between the observations, and were displaced in time to fit the decline feature of the VSNET outbursts for comparison. Because the outburst stage of our $B$ measurement are unknown, we plotted this point at zero time for comparison purposes. The open circles refer to $V$ out-of-eclipse magnitudes measurements of BJS in 1988, when the object was probably also in outburst (see the discussion of Baptista \& Steiner 1991). These points were also aligned to the early decline region of the VSNET outbursts.

The slope of the decline branch of our data in $V$ and $R$ bands is in accordance with that derived from the VSNET data. Our $B$ band measurement is probably representative of the flat-top branch of maximum light of a regular outburst, since the star kept that same brightness level for at least 3 nights. All of our data shown in Fig. 3 are consistent with some stage of the outburst or decline from an outburst of V4140 Sgr in comparison to the observed time scales and amplitudes of VSNET observations. The BJS points could also correspond to early decline phase from outburst maximum. 
The outburst decay time scale seen in the lower panel of Fig. 2 is of order of 1-2 days, much smaller than the time scale of hundreds of days of the transition to the low states of VY Scl-type stars or tens of days seen in fading episodes of some dwarf novae (Kato 2004). In order to determine the decay time scale $\tau_{\mathrm{d}}$ (in days $\mathrm{mag}^{-1}$ ), we fit an exponential function to the $R$ band data of 1992, excluding the times in which the object was in eclipse. The inferred values of $\tau_{\mathrm{d}} \simeq 1.2 \mathrm{~d} \mathrm{mag}^{-1}$ is in agreement with those of SU UMa dwarf novae in a $\log \tau_{\mathrm{d}} \times \log P_{\text {orb }}$ diagram (Warner 1995).

The observed amplitude of the superoutbursts $(\Delta \mathrm{mag} \simeq 2)$ and the expected amplitude of the regular outbursts $(\Delta \operatorname{mag} \simeq 1)$ of V4140 Sgr are in clear disagreement with the typical values for dwarf novae stars. If we consider an outburst amplitude in the range $A_{n}=1-2 \mathrm{mag}$ and a recurrence time interval in the range $T_{n}=80-90$ days, the object falls far away from the expected Kukarkin-Parenago relation in an amplitude versus recurrence time for dwarf novae outbursts (Warner 1995).

\section{Data analysis}

\subsection{The revised orbital parameters}

We found an error in the determination of the binary parameters of BJS, caused by the use of a wrong relation for the width of the white dwarf eclipse (see Fig. 6 of BJS). Here the binary parameters of V4140 Sgr are revised. We adopted the same observed parameters of BJS: a width of the white dwarf eclipse of $\Delta \phi=0.0378 \pm 0.0005$ cycles and a mean duration of the white dwarf ingress/egress feature of $\Delta_{\mathrm{wd}}=0.009 \pm 0.001$ cycles. The value of $\Delta \phi$ derived from our data is the same as above within the uncertainties.

The basis of the photometric binary parameters determination method consists in the measurement of white dwarf contact phases. Detailed descriptions of the method can be found in BJS and Baptista \& Catalán (2000). A Monte Carlo propagation code was used to estimate the errors in the calculated parameters. The values of the input parameters are independently varied according to Gaussian distributions with standard deviations equal to the uncertainty of those parameters. The new results are listed in Table 2 with their 1- $\sigma$ errors. The parameters of BJS are also listed for comparison. $K_{1}$ and $K_{2}$ are the expected values of the primary and secondary star radial velocity semi-amplitude, respectively, as derived from our photometric model.

The primary-secondary mass diagram for V4140 Sgr can be seen in Fig. 4. The cloud of points was obtained from a set of $10^{4}$ trials using the Monte Carlo code. The highest concentration of points indicates the region of most probable solutions. BJS found $R_{2}=0.12 R_{\odot}$, suggesting that the secondary star was oversized with respect to main sequence stars of same mass. Our revised determination leads to $M_{2}=0.09 M_{\odot}$ and $R_{2}=0.136 R_{\odot}$, consistent with the empirical mass-radius relation of Caillaut \& Patterson (1990) at the $1-\sigma$ level. The revised secondary mass is also consistent with the results of Smith \& Dhillon (1998), who found that the secondary stars of CV are indistinguishable from isolated main sequence stars of same mass.
Table 2. Comparison of the orbital parameters.

\begin{tabular}{lll}
\hline \hline & Present work & BJS (1989) \\
\hline$q$ & $0.125 \pm 0.015$ & $0.15 \pm 0.03$ \\
$i$ & $80.2^{\circ} \pm 0.5^{\circ}$ & $79.4^{\circ} \pm 0.9^{\circ}$ \\
$M_{1} / M_{\odot}$ & $0.73 \pm 0.08$ & $0.44 \pm 0.07$ \\
$M_{2} / M_{\odot}$ & $0.092 \pm 0.016$ & $0.07 \pm 0.02$ \\
$R_{1} / R_{\odot}$ & $0.0108 \pm 0.0008$ & $0.014 \pm 0.002$ \\
$R_{2} / R_{\odot}$ & $0.136 \pm 0.008$ & $0.12 \pm 0.01$ \\
$a / R_{\odot}$ & $0.61 \pm 0.05$ & $0.52 \pm 0.03$ \\
$K_{1}\left(\mathrm{~km} \mathrm{~s}^{-1}\right)$ & $55 \pm 7$ & - \\
$K_{2}\left(\mathrm{~km} \mathrm{~s}^{-1}\right)$ & $442 \pm 15$ & $370 \pm 20$ \\
\hline
\end{tabular}

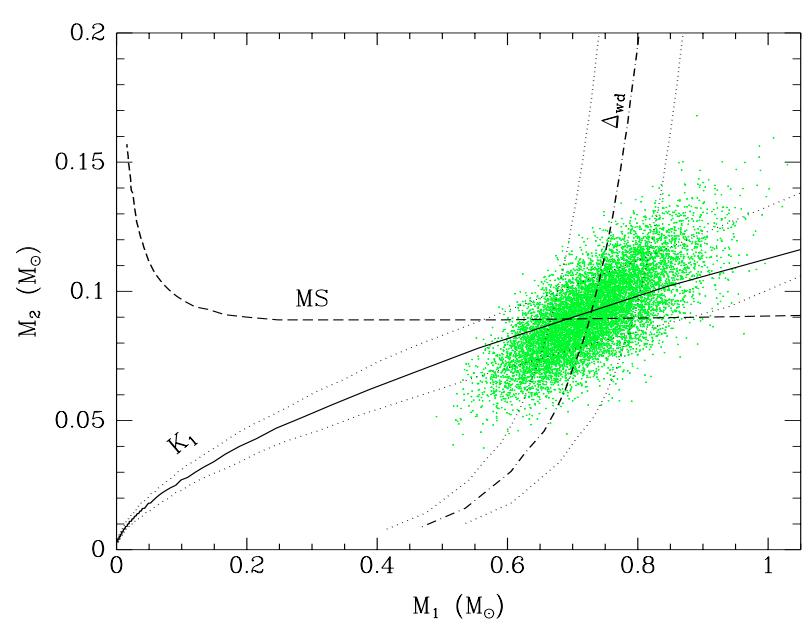

Fig. 4. The primary-secondary mass diagram for V4140 Sgr. The cloud of points is the result of $10^{4}$ Monte Carlo simulations with the data points. The dashed line labeled as MS depicts the empirical mass-radius relation of Caillaut-Patterson for main sequence stars. The solid line is the relation derived from the primary radial velocity semi-amplitude $K_{1}$ obtained by Mukai et al. (1988) and the dot-dashed line is the $M_{1}(q)$ relation obtained by the measurement of $\Delta_{\mathrm{wd}}$. The dotted lines correspond to the uncertainties at the $1-\sigma$.

The new value of the mass ratio $q\left(=M_{2} / M_{1}\right)$ differs from the previous value by less than $20 \%$, while the difference in the inclination $i$ is less than $1 \%$. The largest change occurs in the primary mass. The previous value of $M_{1}=0.44 M_{\odot}$ was much lower than the observed mean white dwarf mass in CVs below the gap $\left(\overline{M_{1}}=0.69 \pm 0.13 M_{\odot}\right.$, Smith \& Dhillon 1998; $\overline{M_{1}}=0.66 \pm 0.01 M_{\odot}$, Webbink 1990). The revised value of $M_{1}=0.73 M_{\odot}$ is consistent with the mean white dwarf of Smith \& Dhillon (1998). The calculated values of the orbital separation $a$ and the predicted $K_{2}$ increased by $18 \%$. A detailed discussion about the uncertainties affecting the photometric model developed here can be found in BJS.

\subsection{The eclipse maps}

The eclipse mapping method (EMM) is an inverse problem resolution technique that uses the information contained in the shape of the eclipse light curve to reconstruct the surface 
Table 3. Identification of the average light curves used in EMM.

\begin{tabular}{cccl}
\hline \hline Date & Passband & Brightness state & Identification \\
\hline 1998, 1999, 2000* & $B$ & quiescence & Bquies \\
2001* & $B$ & outburst & Bout \\
1991* & $V$ & quiescence & Vquies \\
$1992^{*}$ & $V$ & outburst decline & Vdecl \\
1991* & $R$ & quiescence & Rquies \\
1992 Jul. 27 & $R$ & outburst & Rout \\
1992 Jul. 28 & $R$ & outburst decline & Rdecl \\
\hline
\end{tabular}

* Corresponds to all cycles of the year(s) in that passband (see Table 1 for details).

brightness distribution of the accretion disc through an entropy maximization procedure (Skilling \& Bryan 1984). For details of the method, the reader is referred to Horne (1985), Rutten et al. (1992a), Baptista \& Steiner (1993) and Baptista (2001).

The individual light curves were phase folded according to linear plus sinusoidal ephemeris of Baptista et al. (2003)

$$
\begin{aligned}
T_{\mathrm{mid}}= & \text { BJDD } 2446261.67150+0.0614296757 E \\
& +19 \times 10^{-5} \cos \left[2 \pi\left(\frac{E-3 \times 10^{3}}{41 \times 10^{3}}\right)\right] \mathrm{d} .
\end{aligned}
$$

In order to improve the signal-to-noise ratio and to minimize the influence of the characteristic flickering of CVs, the individual light curves were grouped per band and brightness state to produce average eclipse light curves. For each light curve, the data were divided in phase bins of 0.003 cycles and the median was computed for each bin. The median of the absolute deviations with respect to the median was taken as the corresponding uncertainty on each bin. Table 3 summarizes the data used for the EMM, indicating the dates, passbands and brightness state of each average light curve (the details can be seen in Table 1). The last column lists the label used for further identification of the average light curves.

Out-of-eclipse brightness variations are not accounted for the EMM, which assumes that all brightness variations are due to occultation of parts of the disc by the secondary star. The outof-eclipse brightness variations were removed from the average light curves by fitting a spline function to the phases outside eclipse. The light curve was divided by the fitted spline and the result was scaled to the value of the spline at phase zero. This procedure removes the orbital variations with a minor effect on the eclipse shape itself. To speed the convergence of the EMM, all points outside of phase range $(-0.15,+0.15)$ were removed. This does not affect the analysis since the points ruled out are far from the narrow eclipse of the accretion disc of V4140 Sgr.

The eclipse geometry was defined by the mass ratio $q=$ 0.125 and inclination $i=80.2^{\circ}$ (Section 4.1), which corresponds to a value of $\Delta \phi=0.0378$ cycle for the width of the white dwarf eclipse. As mentioned above, the average light curves were phase folded by Eq. (2), therefore the phase of inferior conjunction is $\phi_{0}=0$. This combination of parameters ensures that the white dwarf is at the centre of the map. A flat grid of $51 \times 51$ pixels centered in the white dwarf with side $2 R_{L_{1}}$ was adopted as eclipse map. For V4140 Sgr, a value of $R_{L_{1}}=0.424 R_{\odot}$ is obtained.
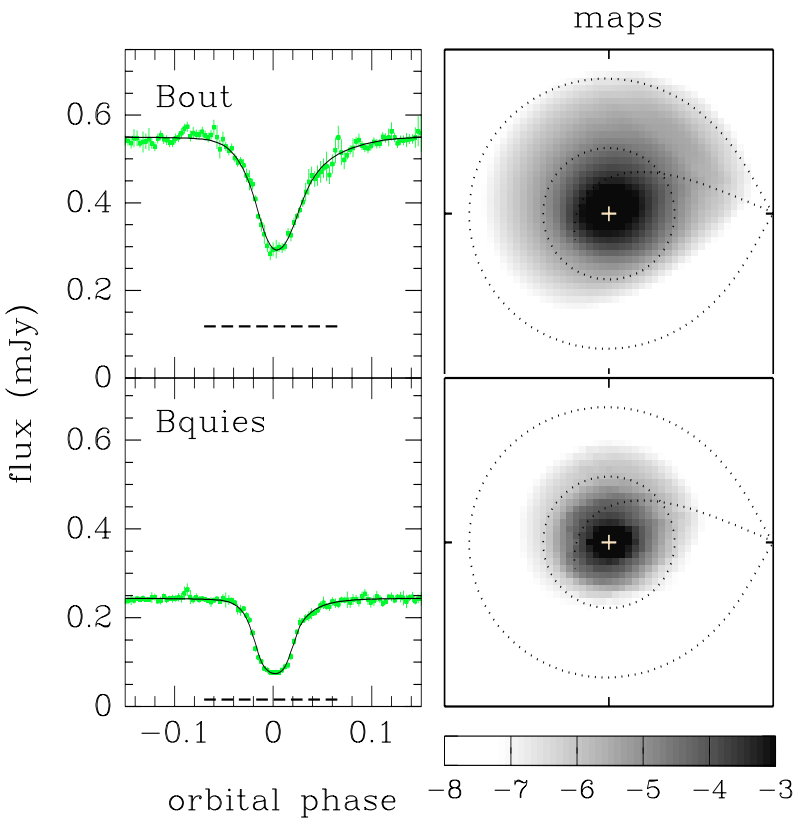

Fig. 5. Left: average light curves of V4140 Sgr in the $B$ band with respective error bars (dots) and the model light curves (solid lines). A dashed line indicates the uneclipsed component in each case. Right: surface brightness distributions of the accretion disc of V4140 Sgr in a logarithmic grey-scale. Brighter regions are indicated in black and the fainter regions in white. A cross marks the centre of the disc. The dotted lines show the Roche lobe, the ballistic stream trajectory (Flannery 1975 ) and a disc radius of $R_{\mathrm{d}}=0.4 R_{L_{1}}$ (see text for explanation). The grey-scale bar corresponds to a linear scale in log of intensity from -8 to -3 .

The average light curves were then analyzed with EMM to reconstruct the surface brightness distribution of the accretion disc of V4140 Sgr and to obtain the additional flux of the uneclipsed component. The latter accounts for all light that is not contained in the eclipse map and can be obtained from the entropy function (Rutten et al. 1992a). A default map of limited azimuthal smearing (Rutten et al. 1992a) was adopted, which is more appropriate for recovering asymmetric structures than the original default of full azimuthal smearing (Baptista et al. 1996). The map are shown in Figs. 5-7. The identification of the average light curves follows the nomenclature of Table 3. The quiescent disc radius was estimated from the position of the bright spot in the $V$ quiescence state map (the centroid of the asymmetry in the Vquies eclipse map) to be $R_{\mathrm{d}} \simeq 0.4 R_{L_{1}}$.

Table 4 summarizes the fractional contribution of the uneclipsed component by passband and by brightness state. The contribution of the uneclipsed component increases significantly in outburst, reaching more than $20 \%$ of the total flux in the $B$ band. The two natural hypotheses to explain the observed increase of the uneclipsed flux during the outburst are the appearance of a wind from the disc and the presence of a flared accretion disc (Baptista \& Catalán 2001).

The average quiescent light curves show narrow, smooth and slightly asymmetric eclipses, which map into smooth brightness distributions with maximum light at disc centre that are slightly brighter at the position of the bright spot at disc rim. During outburst, the eclipses become shallower and more 


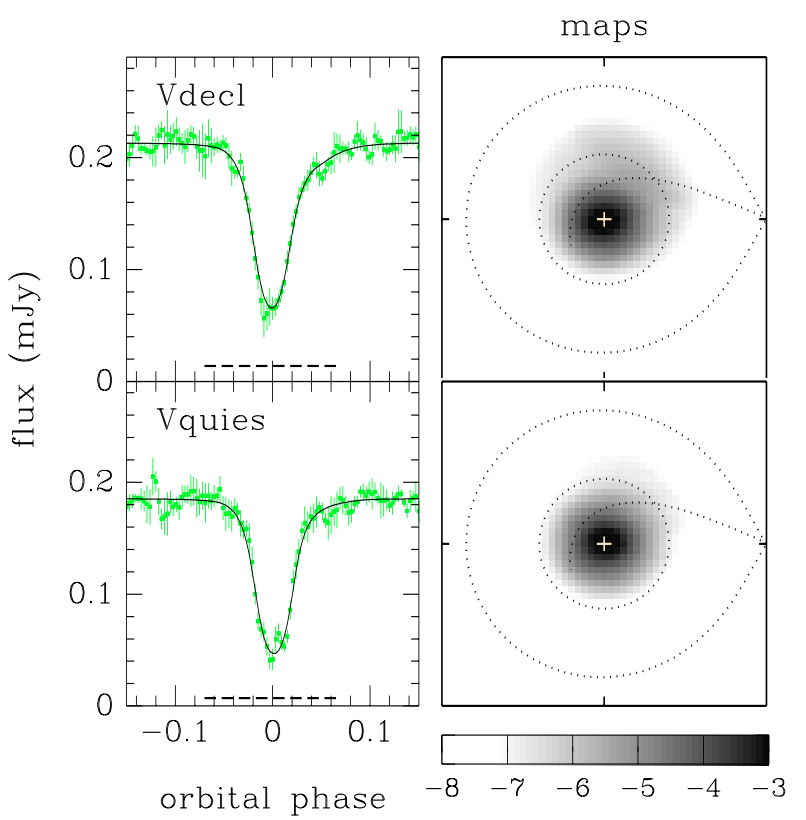

Fig. 6. Left: average light curves of V4140 Sgr in the $V$ band with respective error bars (dots) and the model light curves (solid lines). Right: surface brightness distributions of the accretion disc of V4140 Sgr in a logarithmic grey-scale. The notation is the same as in Fig. 6.

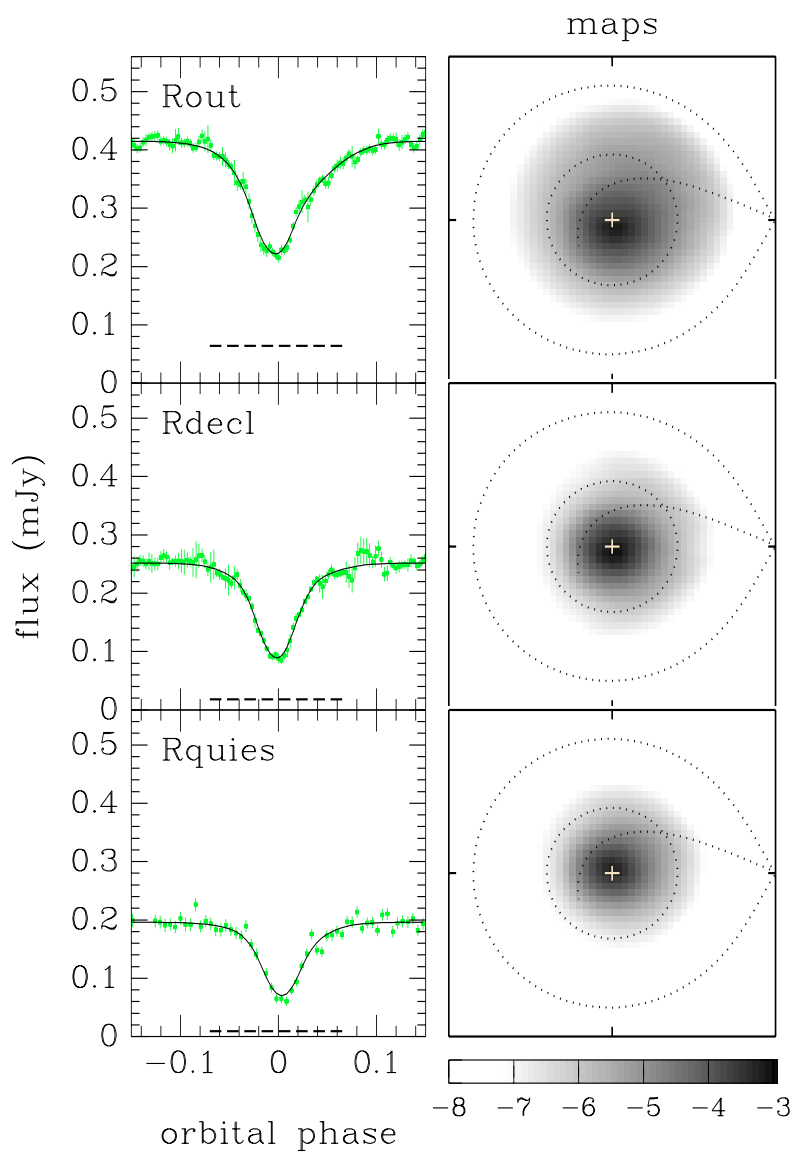

Fig. 7. Left: average light curves of V4140 Sgr in the $R$ band with respective error bars (dots) and the model light curves (solid lines). Right: surface brightness distributions of the accretion disc of V4140 Sgr in a logarithmic grey-scale. The notation is the same as in Fig. 6.
Table 4. Fractional contribution of the uneclipsed component.

\begin{tabular}{cccc}
\hline \hline Passband & \multicolumn{3}{c}{ Brightness state } \\
& outburst & decline & quiescence \\
\hline$B$ & 0.21 & - & 0.07 \\
$V$ & - & 0.07 & 0.04 \\
$R$ & 0.15 & 0.07 & 0.05 \\
\hline
\end{tabular}

asymmetric towards the egress, resulting in eclipse maps with increased brightness in the outer parts of the accretion disc and larger asymmetries at the bright spot position. There is no evidence of a front-to-back brightness contrast in the eclipse maps, which argues against a significant flaring of the accretion disc at this stage ${ }^{3}$. The $V$ band light curve of 1992 (Vdecl, see Table 3) represents a brightness state just after an outburst and may not correspond to the typical quiescent level. Thus, for the following analysis we adopted the light curve and eclipse map of 1991 as representative of the quiescent state in the $V$ band (and labeled as Vquies in consequence).

The uncertainty of each pixel in the eclipse maps was estimated by a bootstrap procedure (Efron 1982). We generated 20 light curves for each average curve identified in Table 3 and applied the EMM to these curves to obtain eclipse maps. The uncertainty of each pixel was taken as the absolute deviations of the sample of 20 bootstrap maps with respect to the intensity of the real map.

\section{Results}

\subsection{A distance estimate}

The quiescent eclipse maps in the $V$ and $R$ bands allow us to estimate the distance if the disc is optically thick (Bruch et al. 1996). This determination is quite similar to the distance estimate of an open cluster, which is done by fitting the standard main sequence relation to the main sequence stars of the cluster in a color-color diagram. However, each map pixel represented in this diagram has the same solid angle, while real stars not. Therefore, for a correct application of the method, a calibration of the magnitude in terms of surface brightness is required.

It is difficult to investigate the emission properties of an accretion disc with a $(B-V) \times(V-R)$ color-color diagram because of the proximity of the optically thick (blackbody and main sequence) and optically thin relations in this diagram. Previous similar studies (Bruch et al. 1996; Baptista et al. 1996) were based on a $(U-B) \times(B-V)$ diagram, in which the optically thin and optically thick emission relations are clearly separated.

The Barnes-Evans empirical relation (Barnes \& Evans 1976; Barnes et al. 1978) relates the surface brightness parameter $F_{V}$ to the color index $(V-R)$, the apparent visual magnitude and the angular diameter. The EMM program yields the intensity of each pixel as seen under the standard condition (a distance of $1 \mathrm{kpc}$, a Roche lobe radius of $1 R_{\odot}$, and a face-on

\footnotetext{
${ }^{3}$ If the disc is flared, the disc side farther away from the secondary star (the "back" side) is made apparently brighter than the disc side closest to the secondary (the "front" side) because it is seen at a lower inclination angle and has larger effective area.
} 
disc, i.e., $\cos i=1)$. Because the $(V-R)$ color index is known for each pixel, the Barnes-Evans relation allows to evaluate its apparent magnitude for an angular diameter calculated under standard conditions. The difference between this value and the value derived directly from the intensities allows us to estimate the distance. Note again that the method assumes an optically thick disc emission. If the disc is optically thin, the inferred distance will be wrong.

The lower panel of Fig. 8 shows the color-magnitude diagram used to calculate the distance to V4140 Sgr. The uncertainty of the points (filled and open squares) are obtained from the uncertainty in the intensities of individual pixels (see Sect. 4.2) and are typically smaller than the plotted symbols. A reddening of $E(B-V)=0.4 \mathrm{mag} \mathrm{kpc}^{-1}$ is estimated for V4140 Sgr from the galactic interstellar extinction contour maps of Lucke (1978). The first two points were discarded from the fit because of the central intensity lowering effect of the maximum entropy method (e.g. Bruch et al. 1996). Assuming an optically thick emission for the disc, a distance of $d=170 \pm 30 \mathrm{pc}$ is obtained. The top panel of Fig. 8 presents the $\chi^{2}$ versus distance diagram used to find the best-fit to the Barnes-Evans relation.

\subsection{Radial temperature distributions}

A basic prediction of the accretion disc theory is that the effective temperature of a steady-state optically thick disc depends on the distance from the disc centre $r$ as

$T_{\mathrm{eff}}^{4}=\frac{3 G M_{1} \dot{M}}{8 \pi \sigma R_{1}^{3}}\left(\frac{r}{R_{1}}\right)^{-3}\left[1-\left(\frac{R_{1}}{r}\right)^{\frac{1}{2}}\right]$

where $\dot{M}$ is the mass transfer rate and the remaining symbols have their usual meaning. This expression is derived from the energy dissipation rate in the disc by unit surface area (Frank et al. 1992).

The intensity maps were converted to blackbody brightness temperature to facilitate its comparison with accretion disc theoretical models. The radial brightness temperature distributions are presented in a logarithmic scale in Figs. 9 and 10. The solid angle used to evaluate the brightness temperature corresponds to a distance of $170 \mathrm{pc}$. For each distribution, steady-state optically thick disc models for different values of $\dot{M}$ (Eq. (3)) and the critical effective temperature of the DI model (Eq. (1)) are plotted for comparison.

It is important to point out here that the brightness temperature is a physical quantity different from the effective temperature given by Eqs. (3) and (1). The relation between those two quantities is non-trivial, as discussed by Baptista et al. (1996), and can only be properly obtained by constructing selfconsistent models of the vertical structure of the disc. This is beyond the scope of this work. On the other hand, the comparison with Eqs. (3) and (1) can be satisfactorily applied to the optically thick disc regions, where the brightness temperature is expected to be close to the effective temperature. Therefore, our analysis is only a good indicator of the disc behavior if the optically thick disc emission hypothesis is valid.

The temperatures range from $3000 \mathrm{~K}$ in the outer disc regions $\left(r \sim 0.4 R_{L_{1}}\right)$ to $\sim 6000 \mathrm{~K}$ in the inner disc regions
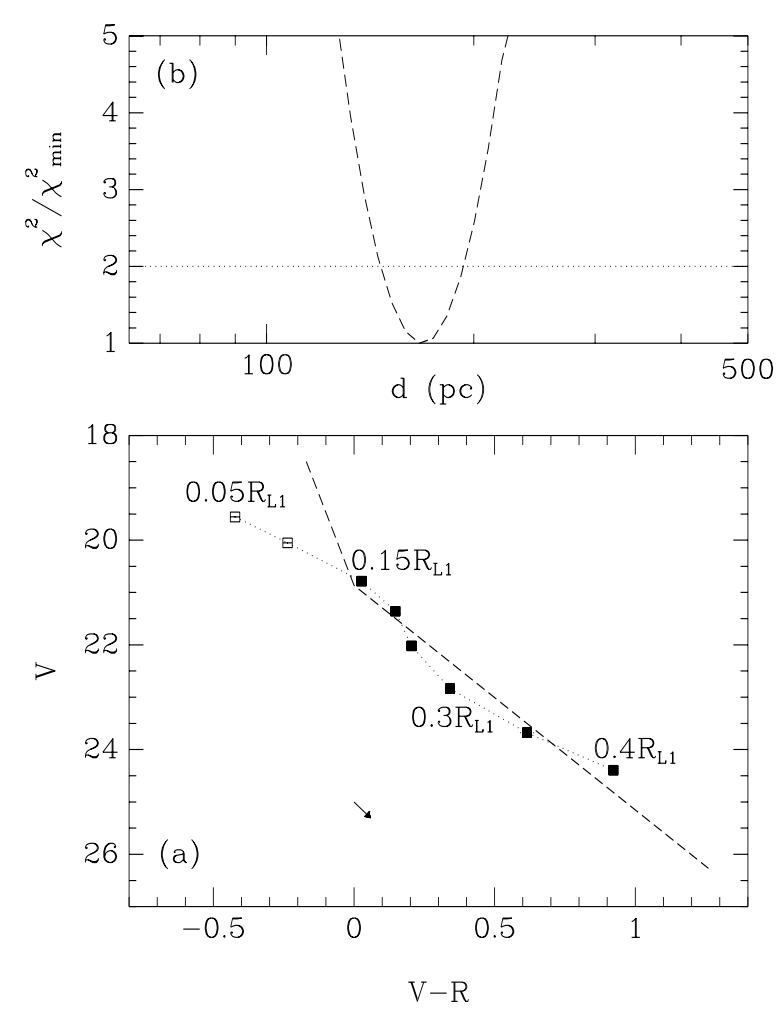

Fig. 8. a) The color-magnitude diagram used to calculate the distance to V4140 Sgr. Filled squares show the average color and magnitude of pixels in radial bins of width $0.05 R_{L_{1}}$ of the $V$ and $R$ eclipse maps in quiescence (Vquies and Rquies maps), starting from disc centre $\left(0.05 R_{L_{1}}\right)$ to the outer regions of the disc $\left(0.4 R_{L_{1}}\right)$. The dashed line is the best fit to the Barnes-Evans relation (Barnes et al. 1978), and corresponds to a vertical displacement of $\Delta V=0.1 \mathrm{mag}$ of the data points. The first two points (open squares) were discarded from the fit because of the central intensity lowering effect of the maximum entropy method. A reddening vector corresponding to $d=170 \pm 30 \mathrm{pc}$ $(E(B-V)=0.07)$ is shown for reference. b) A $\chi^{2}$ versus distance (or versus vertical displacement $\Delta V$ ) diagram is used to find the best fit to the Barnes-Evans relation. The dotted line indicates the $\Delta \chi^{2}$ level used to estimate the error in $d$.

$\left(r \sim 0.1 R_{L_{1}}\right)$. The temperature distributions, both in quiescence and in outburst, are flatter than the $T \propto r^{-\frac{3}{4}}$ law expected for optically thick steady-state accretion discs. The derived disc temperatures are systematically lower than the critical temperature $T_{\text {crit }}$ below which the disc gas should remain to allow outbursts (according to the DI model), both in quiescence and in outburst. The outburst occurs mainly with a significant increase in brightness of the intermediate and outer disc regions, the temperature of which remain below $T_{\text {crit }}-$ in contrast with the expectation of the DI model.

We estimate the velocity of the inward traveling cooling wave along the decline by tracing the radial position of a reference, arbitrary intensity level in the radial intensity distribution. The reference intensity level corresponds to a brightness temperature of $2950 \mathrm{~K}$. Vertical ticks mark the radial position of the reference intensity level along decline in Fig. 10. Combining the change in position $\Delta r$ with the time interval between these eclipse maps (Rout and Rdecl maps), we infer a cooling wave velocity of $\simeq 0.5 \mathrm{~km} \mathrm{~s}^{-1}$, of the same order of the 


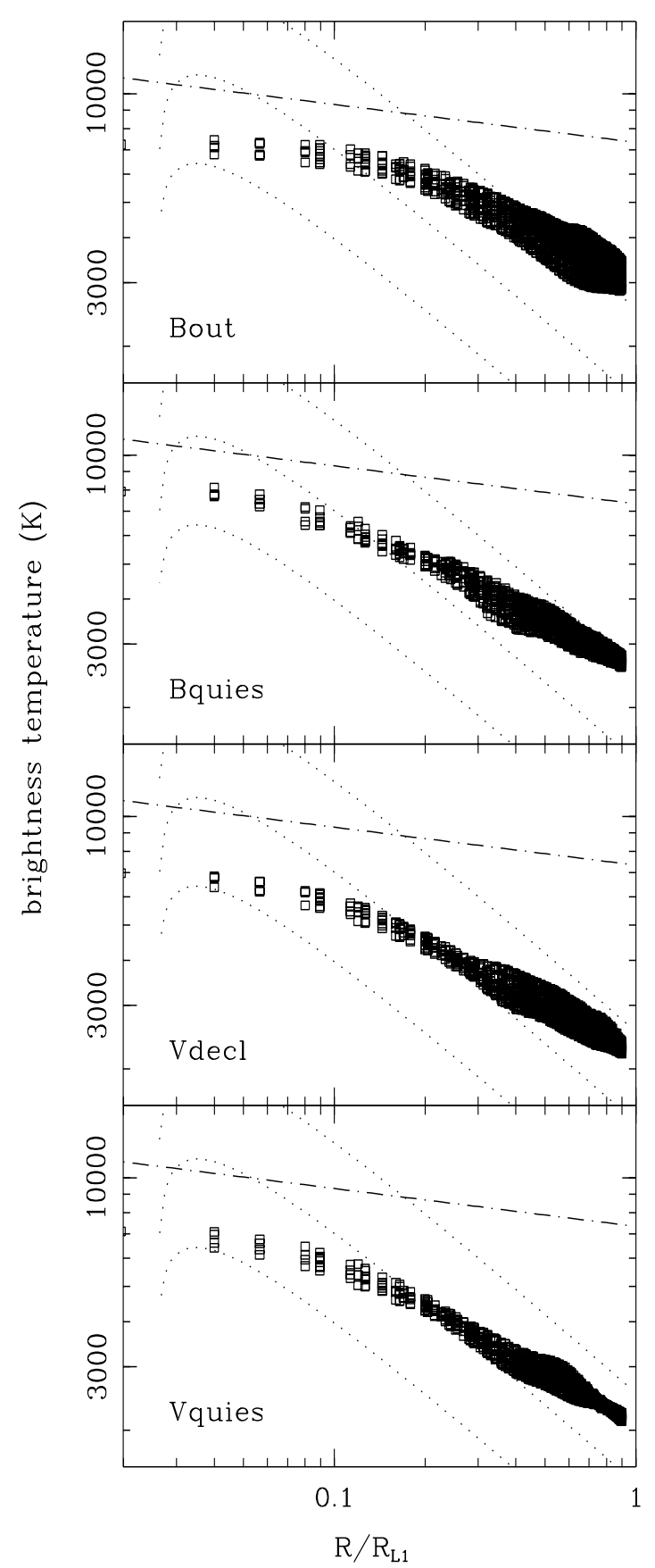

Fig. 9. Radial brightness temperature distributions for the $B$ and $V$ disc maps in outburst, decline and quiescence states. The individual pixels of the disc maps are shown as open squares. Dotted lines correspond to steady-state optically thick disc models for mass accretion rates of $10^{-10}, 10^{-11}$ and $10^{-12} M_{\odot} \mathrm{yr}^{-1}$, from top to bottom respectively. A dotdashed line shows the critical effective temperature $T_{\text {crit }}$ below which the disc gas should remain to allow the thermal instability, and above which the gas should stay while in outburst (e.g. Warner 1995).

velocity estimated for the cooling wave in EX Dra (Baptista \& Catalán 2001).

Assuming a steady-state disc model, the inferred mass accretion rate is $10^{-11.6 \pm 0.3} M_{\odot} \mathrm{yr}^{-1}$ at $r=0.1 R_{L_{1}}$ and $10^{-10.8 \pm 0.2} M_{\odot} \mathrm{yr}^{-1}$ at $r=0.4 R_{L_{1}}$ for the quiescent state. At the decline from outburst maximum, the rate is $10^{-11.6 \pm 0.2} M_{\odot} \mathrm{yr}^{-1}$ at $r=0.1 R_{L_{1}}$ and $10^{-10.9 \pm 0.2} M_{\odot} \mathrm{yr}^{-1}$ at $r=0.4 R_{L_{1}}$.

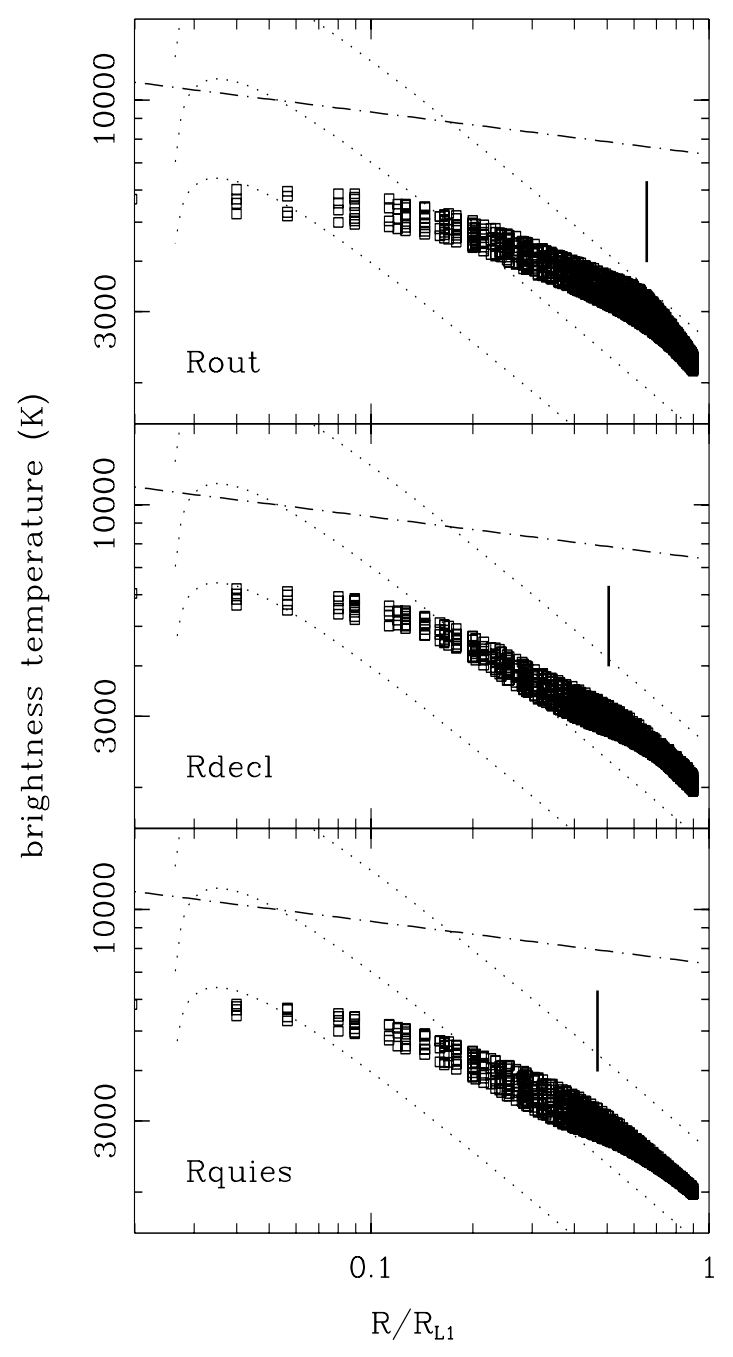

Fig. 10. Radial brightness temperature distributions for the $R$ disc maps in outburst, decline and quiescence states. The notation is the same as in Fig. 9. The vertical ticks in the panel are used to estimate a cooling wave velocity of $\sim 0.5 \mathrm{~km} \mathrm{~s}^{-1}$.

For these two brightness states, the calculated rates are the same at all passbands under the uncertainties. For the outburst state, the inferred values of $\dot{M}$ are systematically different for the $B$ and $R$ bands, probaly reflecting the fact that they correspond to different outbursts. The mass accretion rates inferred from Bout are $10^{-11.4 \pm 0.2} M_{\odot} \mathrm{yr}^{-1}\left(r=0.1 R_{L_{1}}\right)$ and $10^{-10.4 \pm 0.2} M_{\odot} \mathrm{yr}^{-1}\left(r=0.4 R_{L_{1}}\right)$, where for Rout the numbers are $10^{-11.7 \pm 0.2} M_{\odot} \mathrm{yr}^{-1}\left(r=0.1 R_{L_{1}}\right)$ and $10^{-10.7 \pm 0.2} M_{\odot} \mathrm{yr}^{-1}$ $\left(r=0.4 R_{L_{1}}\right)$. Since the accretion disc is seemingly not in a stationary regime during outburst, these mass accretion rates are only illustrative and should be looked at with some reservation.

\section{Discussion}

V4140 Sgr shows a peculiar behavior when its quiescent and outburst radial temperature distributions are compared with those of other dwarf novae of similar orbital period. In order to quantify the differences, we fitted a relation of the type $T_{\mathrm{b}}(r) \propto r^{-\beta}$ to the radial temperature distributions in the range $0.1-0.3 R_{L_{1}}$ in radius. We also computed $\beta$ values from the 
observed $T_{\mathrm{b}}(r)$ distributions of other dwarf novae for comparison. For $Z$ Cha we find $\beta \simeq 0.2$ in quiescence (Wood 1990) and $\beta \simeq 0.8$ in outburst (Horne \& Cook 1985). For OY Car we find values of $<0.1$ and $\sim 0.7$, respectively for quiescence (Wood 1990) and outburst (Rutten et al. 1992b). For V4140 Sgr we obtain $\beta=0.36 \pm 0.02$ for the quiescent state and for the decline from outburst. From the radial brightness distributions in outburst we obtain $\beta=0.25 \pm 0.03$.

Comparing these values of $\beta$, we see that the radial brightness distribution of $\mathrm{Z}$ Cha and OY Car is flat in quiescence and closely follows the $T \propto r^{-\frac{3}{4}}$ law of optically thick steadystate disc in outburst (Horne \& Cook 1985; Wood et al. 1986; Wood et al. 1989; Wood 1990; Rutten et al. 1992b). In contrast, the radial temperature distribution of V4140 Sgr is clearly steeper than those of OY Car and Z Cha in quiescence, and becomes much flatter than that of those stars in outburst. In Z Cha and OY Car the outbursts occur with a significant increase in the relative brightness of the inner disc regions, whereas in V4140 Sgr the outbursts reflect the increase in brightness of only the outer disc regions. The small amplitude and the flat temperature distribution of V4140 Sgr during outburst may indicate a different physical origin when compared with "normal" dwarf novae outburst observed e.g. in Z Cha, OY Car or SS Cyg.

Our distance estimate relies on the arguable assumption that the quiescent disc of V4140 Sgr is optically thick. The inferred disc temperatures depend on the assumed distance. We investigated the effect of the distance on the derived brightness distributions, trying to determine at which distance the binary should be in order to make the brightness temperatures in the outbursting outer disc regions higher than $T_{\text {crit }}$.

This only occurs if the distance is made larger than about $\sim 800$ pc. However, at this large distance the radial temperature distribution in quiescence becomes steeper and closely follows the $T \propto r^{-\frac{3}{4}}$ law, implying that the quiescent disc of V4140 Sgr should be in a high viscosity, steady-state regime. In other words, the only way to remove the inconsistency regarding the lower-than-expected temperatures in the outer disc is by making the dwarf nova so bright that it becomes a novalike variable. Figure 11 illustrate this by comparing the derived radial temperature distributions in quiescence and outburst for distances of $170 \mathrm{pc}$ and $900 \mathrm{pc}$.

Thus, this exercise indicates that the small outburst seen in the light curve of V4140 Sgr are very likely not caused by disc instabilities according to the DI model. An alternative, plausible explanation is that the outbursts of V4140 Sgr are episodes of increased mass transfer from the companion star onto an accretion disc permanently in a high viscosity regime. In this scenario, the low amplitude of the outbursts of V4140 Sgr would be a consequence of its quiescent, high-viscosity inner disc being hotter and brighter than those of "normal" dwarf novae of similar orbital period (e.g. Z Cha and OY Car). The comparison of eclipse shapes in quiescence supports this idea. The pronounced orbital hump and the double-stepped eclipses of $Z$ Cha and OY Car reflect the fact that the quiescent disc is relatively faint in comparison to the white dwarf and bright spot in these stars. On the other hand, the white dwarf, bright spot and orbital hump features are much less conspicuous in the eclipses

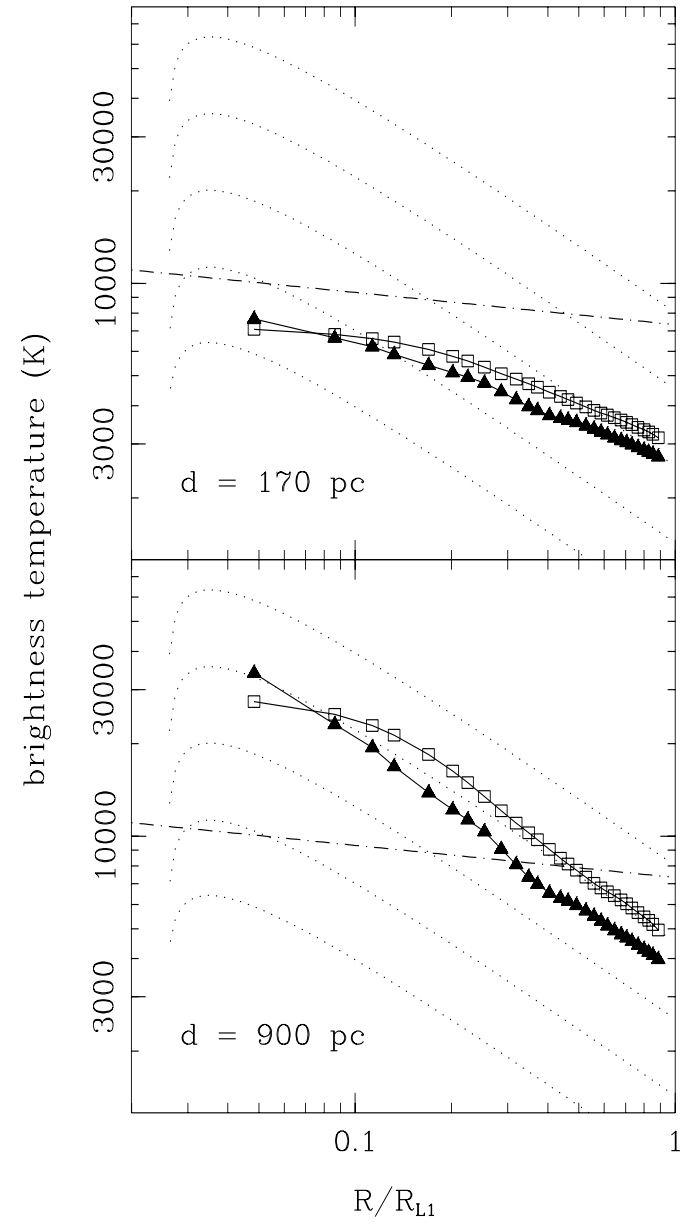

Fig. 11. Average radial brightness temperature distributions for the $B$ disc maps in outburst and quiescent states calculated for distances of $170 \mathrm{pc}$ (top panel) and $900 \mathrm{pc}$ (lower panel). For each panel, the points represents the mean temperature values computed in radius bins of $0.03 R_{L_{1}}$. Solid triangle and open squares correspond to the quiescence and outburst states (Bquies and Bout), respectively. Dotted lines correspond to steady-state optically thick disc models for mass accretion rates of $10^{-8}, 10^{-9}, 10^{-10}, 10^{-11}$ and $10^{-12} M_{\odot} \mathrm{yr}^{-1}$, from top to bottom respectively. A dot-dashed line shows the critical effective temperature $T_{\text {crit }}$ below which the disc gas should remain to allow the thermal instability, and above which the gas should stay while in outburst (e.g. Warner 1995).

of V4140 Sgr (Fig. 3; see also Fig. 4 of BJS), indicating that the quiescent disc is a relatively much more important source of light than in Z Cha or OY Car.

V4140 Sgr may be a member of an increasing group of dwarf novae the behavior of which cannot be explained by the DI model, together with EX Dra (Baptista \& Catalán 2001), EX Hya (Hellier et al. 2000) and V2051 Oph (Baptista \& Bortoletto 2004).

\section{Conclusions}

The main results of our photometric study of V4140 Sgr can be summarized as follows:

(i) Our observations caught V4140 Sgr in the decline from an outburst in 1992 July and again in outburst in 
2001 July. Further observations of a superoutburst of V4140 Sgr in 2004 by amateur astronomers confirms its SU UMa-type dwarf nova classification. The observed outburst duration is 5-10 days and the time interval between outbursts is 80-90 days.

(ii) The observed amplitudes of the superoutbursts $(\Delta \mathrm{mag} \simeq$ $2)$ and of the regular outbursts $(\Delta \mathrm{mag} \simeq 1)$ of V4140 Sgr are remarkably low in comparison with the typical values for dwarf novae stars. Using the observed outburst amplitude and recurrence time interval, the object lies far away from the expected Kukarkin-Parenago relation.

(iii) The binary parameters were revised. The new primary mass $M_{1}$ is consistent with the observed mean white dwarf mass in CVs, and the new secondary mass $M_{2}$ is comparable to that of main sequence stars of same mass and radius.

(iv) A color-magnitude diagram was used to estimate the distance to V4140 Sgr, with a method similar to that used to constrain the distance to open clusters. Assuming an optically thick emission for the disc, a distance of $170 \pm 30 \mathrm{pc}$ is obtained.

(v) The radial brightness temperature distribution range from $3000 \mathrm{~K}$ in the outer disc regions $\left(r \sim 0.4 R_{L_{1}}\right)$ to $\sim 6000 \mathrm{~K}$ in the inner disc regions $\left(r \sim 0.1 R_{L_{1}}\right)$. The temperature distributions, both in quiescence and in outburst, are flatter than the $T \propto r^{-\frac{3}{4}}$ law expected for an optically thick steady-state accretion disc.

(vi) The derived disc temperatures are systematically lower than the critical temperature $T_{\text {crit }}$ below which the disc gas should remain to allow outburst, both in quiescence and in outburst. The small outburst amplitudes and the flat temperature distributions may indicate that the outbursts are likely not caused by disc instabilities according to the DI model.

(vii) V4140 Sgr shows a peculiar behavior when compared to those of other dwarf novae with similar short orbital period. We suggest that the cause of the small amplitude outbursts of V4140 Sgr are episodes of enhanced mass transfer rate from the secondary star onto a disc in a permanent high-viscosity regime.

Acknowledgements. We thank Christian Knigge for calling our attention to the error in the determination of the binary parameters of V4140 Sgr and an anonymous referee for useful comments that improved the presentation of the paper. In this research we have used, and acknowledge with thanks, data from VSNET, which are based on observations collected by variable star observers worldwide. This work was partially supported by $\mathrm{CNPq} / \mathrm{Brazil}$ through research grant 62.0053/01-1 - PADCT III/Milênio. BWB acknowledges financial support from CAPES/Brazil. RB acknowledges financial support from CNPq through grant 300.354/96-7.

\section{References}

Baptista, R. 2001, Astrotomography, Indirect Imaging Methods in Observational Astronomy (Springer-Verlag), Lecture Notes in Physics, 573, 307

Baptista, R., Borges, B. W., Bond, H. E., et al. 2003, MNRAS, 345, 889
Baptista, R., \& Bortoletto, A. 2004, AJ, 128, 411

Baptista, R., \& Catalán, M. S. 2000, MNRAS, 316, 529

Baptista, R., \& Catalán, M. S. 2001, MNRAS, 324, 599

Baptista, R., Jablonski, F. J., \& Steiner, J. E. 1989, MNRAS, 241, 631 (BJS)

Baptista, R., \& Steiner, J. E. 1991, A\&A, 249, 284

Baptista, R., \& Steiner, J. E. 1993, A\&A, 277, 331

Baptista, R., Steiner, J. E., \& Horne, K. 1996, MNRAS, 282, 99

Barnes, T. G., \& Evans, D. S. 1976, MNRAS, 174, 489

Barnes, T. G., Evans, D. S., \& Moffett, T. J. 1978, MNRAS, 183, 285

Bruch, A., Beele, D., \& Baptista, R. 1996, A\&A, 306, 151

Buat-Ménard, V., Hameury, J.-M., \& Lasota, J.-P. 2001, A\&A, 369, 925

Caillault, J., \& Patterson, J. 1990, AJ, 100, 825

Cannizzo, J. H. 1993, Accretion Disks in Compact Stellar Systems, ed. J. C. Wheeler (World Sci. Publ. Co.), 6

Downes, R. A., Webbink, R. F., Shara, M. M., et al. 2001, PASP, 113, 764

Efron, B. 1982, The Jackknife, the Bootstrap and other resampling plans, CBMS-NSF Regional Conference Series in Applied Mathematics, Society for Industrial and Applied Mathematics (SIAM)

Flannery, B. P. 1975, MNRAS, 170, 325

Frank, J., King, A. R., \& Raine, D. J. 1992, Accretion Power in Astrophyiscs (Cambridge University Press), 2nd ed.

Gontikakis, C., \& Hameury, J.-M. 1993, A\&A, 271, 118

Graham, J. A. 1982, PASP, 94, 244

Harris, W. E., Fitzgerald, M. P., \& Reed, B. C. 1981, PASP, 93, 507

Hellier, C., Kemp, J., Naylor, T., et al. 2000, MNRAS, 313, 703

Horne, K. 1985, MNRAS, 213, 129

Horne, K., \& Cook, M. C. 1985, MNRAS, 214, 307

Jablonski, F., \& Steiner, J. E. 1987, ApJ, 313, 376

Jablonski, F., Baptista, R., Barroso, J., et al. 1994, PASP, 106, 1172

Kato, T. 2004, PASJ, 56, 55

Kato, T., Uemura, M., Ishioka, R., et al. 2004, PASJ, 56, 1

King, A. R., \& Cannizzo, J. K. 1998, ApJ, 499, 348

Lamla, E. 1982, Numerical Data and Functional Relationships in Science and Technology, Landolt-Börnstein New Series, Vol. 2 (Springer-Verlag)

Lasota, J.-P. 2001, New A Rev., 45, 449

Lucke, P. B. 1978, A\&A, 64, 367

Mukai, K., Corbet, R. H. D., \& Smale, A. P. 1988, MNRAS, 234, 291

Rutten, R. G. M., van Paradijs, J., \& Tinbergen, J. 1992a, A\&A, 260, 213

Rutten, R. G. M., Kuulkers, E., Vogt, N., \& van Paradijs, J. 1992b, A\&A, 265, 159

Schreiber, M. R., Gänsicke, B. T., \& Hessman, F. V. 2000, A\&A, 358, 221

Schreiber, M. R., Hameury, J.-M., \& Lasota, J.-P. 2003, A\&A, 410, 239

Skilling, J., \& Bryan, R. K. 1984, MNRAS, 211, 111

Smak, J. 2000, New A Rev., 44, 171

Smith, D. A., \& Dhillon, V. S. 1998, MNRAS, 301, 767

Stone, R. P. S., \& Baldwin, J. A. 1983, MNRAS, 204, 347

Uemura, M. 2004, VSNET Campaign [vsnet-campaign-dn 4382]

Warner, B. 1995, Cataclysmic Variable Stars, Cambridge Astrophysics Series (Cambridge University Press)

Webbink, R. F. 1990, Accretion-Powered Compact Binaries (Cambridge University Press), 177

Wood, J. H. 1990, MNRAS, 243, 219

Wood, J. H., Horne, K., Berriman, G., et al. 1986, MNRAS, 219, 629

Wood, J. H., Horne, K., Berriman, G., \& Wade, R. A. 1989, ApJ, 341, 974 